\title{
THE KNOWLEDGE, ATIITUDES, AND PRACTICE (KAP) OF THE IRANIAN PUBLIC TOWARDS COVID-19: A SYSTEMATIC REVIEW
}

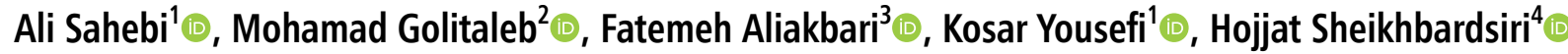 \\ ${ }^{1}$ Non-Communicable Diseases Research Center, Ilam University of Medical Sciences, Ilam, Iran \\ ${ }^{2}$ Department of Nursing, School of Nursing, Arak University of Medical Sciences, Arak, Iran \\ ${ }^{3}$ Community-Oriented Nursing Midwifery Research Center, Shahrekord University of Medical Sciences, Shahrekord, Iran \\ ${ }^{4}$ Health in Disasters and Emergencies Research Center, Institute for Futures Studies in Health, Kerman University of Medical Sciences, Kerman, Iran
}

\begin{abstract}
INTRODUCTION: COVID-19 is an extremely contagious disease that has led to a global pandemic. Control of this pandemic requires community awareness and adherence to preventive behaviors. The aim of this review is to investigate the knowledge, attitudes, and practices (KAP) of the Iranian public towards the COVID-19 pandemic.
\end{abstract}

MATERIAL AND METHODS: This review was conducted according to the PRISMA guidelines. Databases including Magiran, SID, ISC, Scopus, PubMed, Web of Science, Cochrane, ProQuest, Science Direct, and Google Scholar were searched for literature. The searches were conducted in both Farsi and English without any time limit until the end of December 2020. The research question and inclusion and exclusion criteria were designed according to the PICO. For qualifying studies, the NOS checklist was used. We used narrative synthesis for synthesizing the entered studies.

RESULTS: 138 studies were found during the initial search, of which 13 were systematically reviewed. All studies identified had a cross-sectional design and used researcher-designed tools for data collection. Overall, 11111 people (4900 men and 6211 women) from the public population of Iran were included.

CONCLUSIONS: The majority of Iranians studied had appropriate (KAP) regarding COVID-19 precautions. Policymakers should continue public health education measures.

KEY WORDS: general population, knowledge, attitude, practice, systematic review, COVID-19

Disaster Emerg Med J

\section{INTRODUCTION}

Coronavirus disease 2019 (COVID-19) was first reported in Wuhan, China. The disease has since led to a global pandemic with widespread economic, social, and health consequences [1]. One of the important features of COVID-19 is its high transmissibility, necessitating adherence to strict personal and social hygiene [2]. The rapid spread of the virus has overwhelmed the health care infrastructures of many countries [3]. As of January 26, 2021, over 99 million people have been infected with the virus globally, and over 2 million have died [4]. Although the majority of deaths and infections occur in individuals over 50 years of age and those with

ADDRESS FOR CORRESPONDENCE:

Hojjat Sheikhbardsiri, Health in Disasters and Emergencies Research Center, Institute for Futures Studies in Health, Kerman University of Medical Sciences, Kerman, Iran

e-mail: hojat.sheikhbardsiri@gmail.com 
underlying chronic diseases, the risk of contracting the infection extends to the whole population. This highlights the importance of preventive measures for everyone, as asymptomatic carriers can transmit the disease to high-risk individuals [5]. Promoting physical distancing, mask-wearing, and hand hygiene are basic policies that health officials can employ to control the epidemic [6]. The World Health Organization has declared regular hand washing, observing respiratory hygiene, keeping proper physical distance, and avoiding handshakes and hugs as important behaviors to prevent the spread of the disease [7].

Studies have shown that perceived knowledge, attitude, and threat are important predictors of adherence to health behaviors [8]. As there is no definitive treatment for COVID-19, the best way to prevent the spread of this disease is to implement and promote preventive measures (while enrolling in vaccination campaigns). Currently, the most important strategies to control the pandemic, beyond vaccination, include early diagnosis, contact tracing, and quarantine. Another important measure is adherence to personal protective health measures by all members of society. As such, the population should be adequately aware of these measures to plausibly control the spread of the disease [9]. Promoting improved knowledge, a supportive attitude, and compliant behavior among the public, creates an environment of adherence of the public to health instructions aimed at preventing the spread of the disease $[9,10]$. Experiences from the severe acute respiratory syndrome (SARS) outbreak in 2003 and the Middle East respiratory syndrome (MERS) in 2012 demonstrated that inadequate knowledge and negative attitudes can exaggerate the fear of disease and lead to stigmatization of patients [10, 11]. This fear can itself compromise the preventive measures aiming to control the disease. Therefore, it is imperative to improve the awareness and attitudes of health care workers and the public [10, 11]. Insufficient knowledge and negative attitudes can lead to inappropriate practices and increase the spread of this infection [12]. Thus, a key step to control the pandemic is to provide effective instructions to increase public knowledge about the symptoms, prevention strategies, and transmission routes of COVID-19 [10, 13].

Our review aimed to investigate the KAP of Iran's public population about COVID-19. Our findings are expected to help health managers and policymakers to understand public perceptions and implement appropriate infection control policies.

\section{MATERIAL AND METHODS}

The present study was conducted according to the Preferred Reporting Items for Systematic Reviews and Meta-analyses (PRISMA) guidelines [14]. The study protocol was registered in the International Prospective Register of Systematic Reviews (PROSPERO) under the code CRD42021238983.

\section{Search strategy}

Databases of SID, Magiran, ISC, Scopus, PubMed, Web of Science, Cochrane, ProQuest, Science Direct, and Google Scholar were searched using valid English keywords and their Farsi equivalents including knowledge, attitude, opinion, performance, practice, function, "2019 novel coronavirus disease", COVID-19, "COVID-19 pandemic", "SARS-CoV-2 infection", "COVID-19 virus disease", "2019 novel coronavirus infection", "2019-nCoV infection", "Coronavirus disease 2019", "2019-nCoV disease", "COVID-19 virus infection", and Iran. Syntax search was used for this purpose. Initially, using Boolean operators, keywords, and search fields, a syntax search was designed for PubMed. For other databases, the search strategy was defined according to the syntax search developed for PubMed. The searches were conducted in both Farsi and English without any time limit up to December 2020. An example of the search strategy in PubMed has been provided below, and search strategies in other databases have been noted in Appendix 1???

\section{Eligibility criteria}

The research question and inclusion and exclusion criteria were designed according to the PICO principle: participants (Iranian public), Interventions/exposure (COVID-19), comparison (any), Outcome (KAP) Studies that evaluated KAP in other countries, and infectious diseases other than COVID-19 were excluded from the study. All types of studies like observational, interventional, review, and letter to the editor are included.

\section{Study selection}

After the preliminary search in the databases, 138 articles were entered into EndNote X7 software (Clarivate, London, United Kingdom). First, duplicate articles were removed, and then 92 articles 


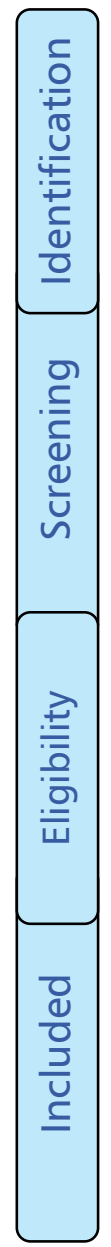

Records identified in database searching $(n=136)$
Additional records identified in other resources $(n=2)$

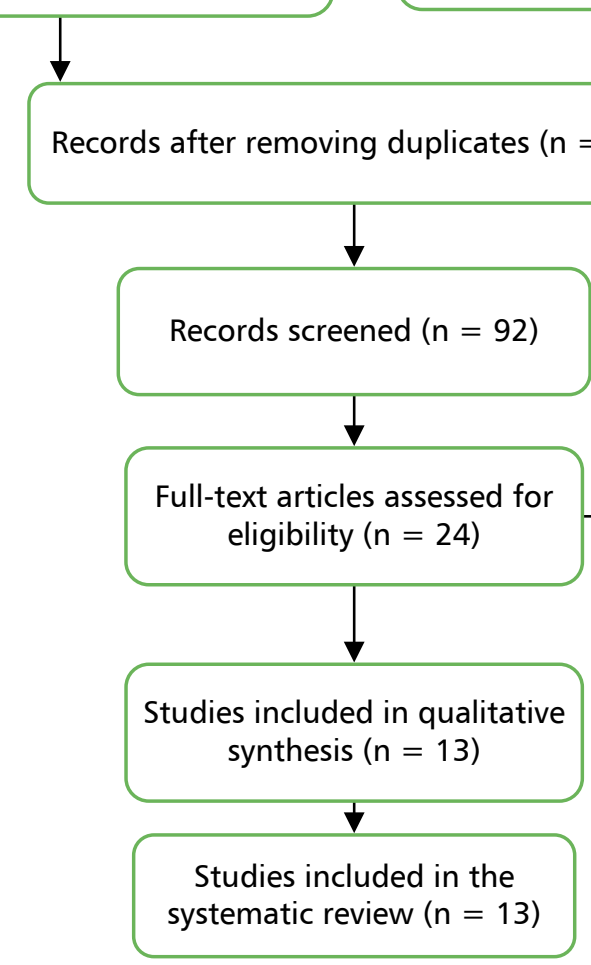

Records excluded $(n=68)$

Full-text articles excluded with reasons $(n=11)$

FIGURE 1. Study flowchart based on the PRISMA guideline

were screened. Subsequently, 24 potentially related studies were selected and reviewed independently in detail by 2 of the researchers (AS, MG). Ultimately, 13 studies were selected for quality assessment.

\section{Qualification and data extraction}

We used The Newcastle-Ottawa Scale (NOS) scale to assess the quality of the studies. NOS scale was used to evaluate the quality of studies. Two authors, AS and $M G$, used this scale independently to assess the methodological quality, scale (comparability), and outcome of each study, and if there was any disagreement between them, a third author opinion was used to make the agreement, and a score of 7 out of 10 was considered as the good quality of the studies. In this research, studies that gain scores seven and higher were included [15], because different methods and scales were used in studies to report the results, and there was a high heterogeneity, so it was not possible to perform meta-analysis on them, so we used narrative synthesis to summarize the research findings. A researcher-designed checklist was used to record the required data including title, first author, location, the total number of subjects, number of subjects by gender, and study findings.

\section{RESULTS}

In the primary search, 138 studies were identified, of which 46 duplicate studies were removed, and 92 studies were further screened. Finally, 13 studies were subjected to quality assessment, and all of them entered the systematic review process. Figure 1 outlines the steps of study selection.

Overall, 11111 Iranians (4 900 men and 6211 women) were included. The methodology of all the studies was cross-sectional. All studies were of good quality, and based on the consultation of the research team, after the narrative synthesis, it was concluded that the factors affecting KAP include education, information, demographic characteristics, and specific groups. because different 
methods and scales were used in studies to report the results, and there was a high heterogeneity, so it was not possible to perform meta-analysis on them, so we used narrative synthesis to summarize the research findings. Table 1 shows the characteristics and findings of these studies.

\section{DISCUSSION}

Most of the evaluated studies reported that Iranians had appropriate KAP to prevent the spread of COVID-19. Similarly, a review by Puspitasar et al. [29] on the general population, health care workers, and medical students in the United States, the United Kingdom, Italy, Jordan, and China showed that the subjects had good levels of knowledge and performance and were optimistic about COVID-19. The results of another study by Kamali et al. [30] in Iran showed that health care workers had an acceptable level of knowledge and good attitudes and practices regarding COVID-19. These findings along with our observation in the present study indicate appropriate KAP towards COVID-19 in most communities, including the general public, patients, and health care workers. Nevertheless, people's knowledge, attitudes, and behaviors should be improved as much as possible by continuing education campaigns. The results of studies by Moradzadeh [17] and Kalani [28] showed that men's KAP regarding COVID-19 infection needs improvement [11]. Similarly, a study in China showed that men had less knowledge, more negative attitudes, and worse performance than women towards COVID-19 infection control measures [11]. This was in line with the results of our review. The cause of this discrepancy is unclear. To improve men's awareness and preventive behaviors regarding COVID-19, we suggest that health systems actively target men in educational programs. In their study, Shaygannejad et al. [22] showed no significant differences comparing the KAP with chronic neurological problems and healthy individuals. According to Sahraian et al. [25], COVID-19 patients had a good understanding of the disease. A study in India revealed that adults with type 1 diabetes had moderate knowledge, positive attitudes, and appropriate preventive practice towards COVID-19 [31]. Ajay et al. [32] in Pakistan showed that despite having adequate knowledge about COVID-19, diabetes patients had taken inappropriate preventive measures like washing hands frequently, maintaining social distancing, avoiding touching eyes, nose, and mouth, staying home when sick, except to get medical care, covering mouth and nose with bent elbow or tissue when coughing or sneezing and maintaining at least $1 \mathrm{~m}$ or $3 \mathrm{ft}$ distance between yourself and anyone who is coughing or sneezing. Other studies such as one in Ethiopia have shown that a higher level of knowledge is associated with better performance and adherence to preventive measures against COVID-19 [33]. Based on the results of these studies and those of this review, it can be concluded that the levels of knowledge and awareness of patients, especially those with chronic diseases, are appropriate and comparable with that of the general public. Based on the results of most of the reviewed studies, institutions rely heavily on information technologies such as the internet, social media, and text messaging for public education. In one study, Alzoubi et al. [34] investigated the knowledge and information resources of medical and non-medical students at Jordan University regarding COVID-19. They showed that most students had been using the internet, social media, and mass media while medical and post-graduate students had also used scientific papers and websites as their sources of information [34]. A study by Chan et al. [35] showed that social media if used correctly and appropriately, can be one of the most effective methods of communication to disseminate information about the COVID-19 pandemic. This study was performed on the public, considering that the public is less prone to COVID-19 disease than health care workers and may not receive the necessary training, so measures such as timely education can make a change in their KAP. In conclusion, it seems that most people use the internet and social media to obtain COVID-19-related information during this pandemic. Since a majority of the population has access to social media and the Internet, these platforms can be the fastest routes to impart important information during the COVID-19 pandemic. However, illiterate people or those who do not have access to the Internet and social media may miss the information provided via these tools. Therefore, the health system should employ a variety of educational methods, including face-to-face training, traditional news, and information media, to increase public awareness of the disease.

\section{Strengths and limitations}

This is the only systematic review on the KAP of Iranians towards COVID-19. Most studies conducted 


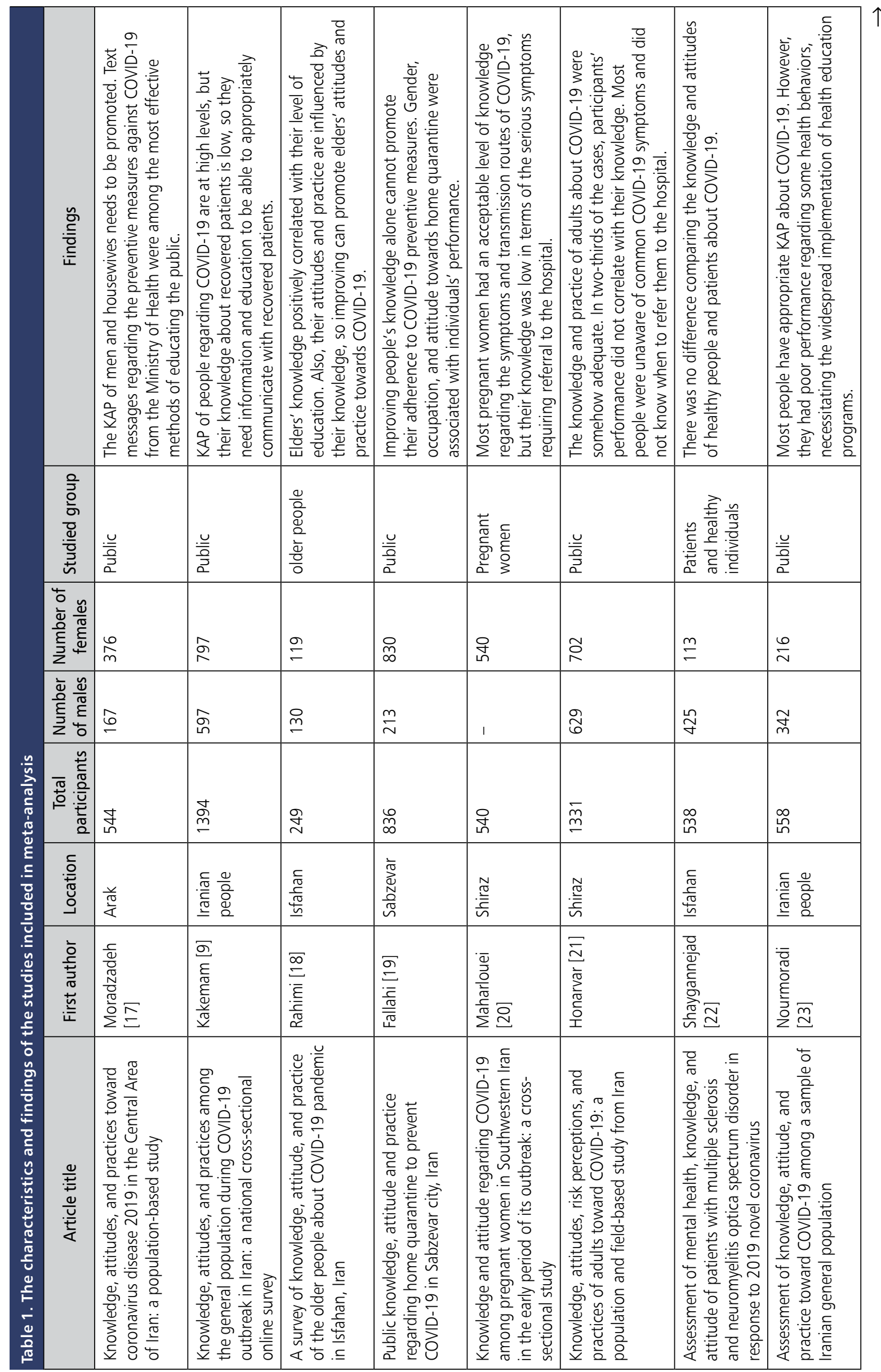




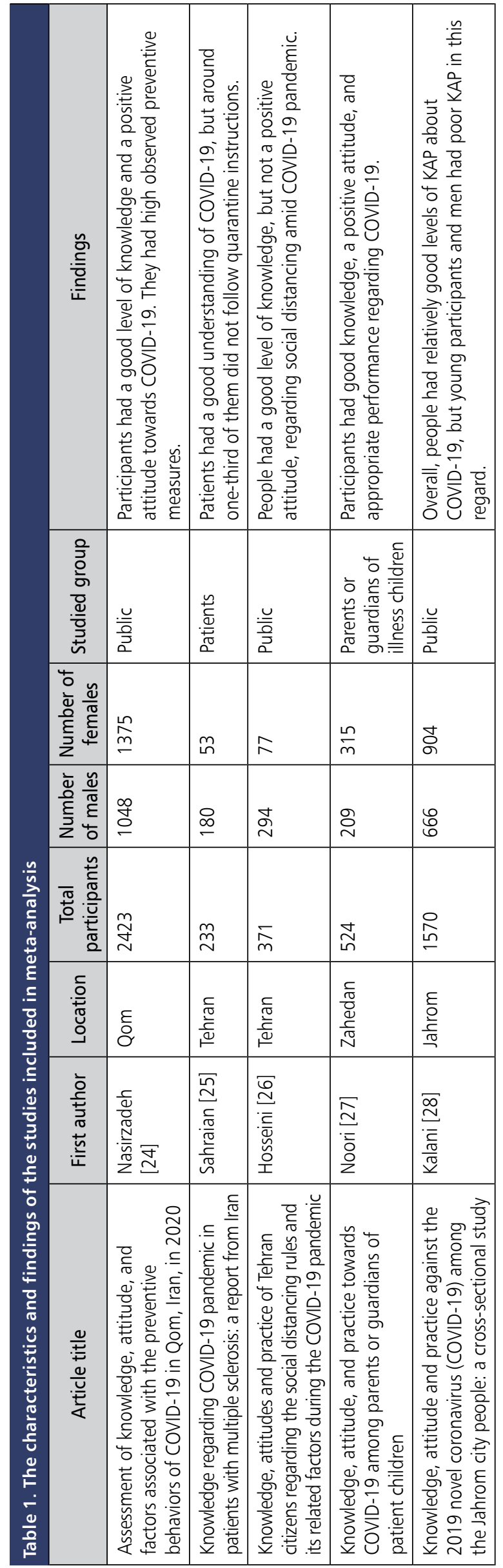

in Iran regarding the KAP of people towards COVID-19 are descriptive and cross-sectional. We did not find any comprehensive studies on this issue. One of the limitations of this review is that some of the evaluated publications did not report the overall levels of KAP of the populations studied. Furthermore, all of the studies were essentially questionnaire-based, which lead to multiple biases and limitations. Finally, the geographic locations of the studies were limited to a few major cities, and thus the data is not representative of the entire country.

\section{CONCLUSIONS}

Based on our systematic review, the public population of Iran has a good level of KAP to prevent the spread of COVID-19. Public information and COVID-19-related training is primarily achieved through new information technology methods. Since higher awareness and knowledge correlate with better performance, policymakers should continue their public health education efforts to control this pandemic.

\section{ACKNOWLEDGMENTS}

The Ethics Committee of Kerman University of Medical Sciences approved this study; A Systematic Review design was employed in 2021, the pajoohan number was 400000861. The authors would like to express their gratitude to the Vice-Chancellor of Research and Technology of Kerman University of Medical Sciences for the financial support of this research.

\section{FUNDING SOURCES}

Kerman University of Medical Sciences financially supported this study.

\section{CONFLICTS OF INTEREST}

The authors declare that they have no competing interests.

\section{REFERENCES}

1. Vardanjani AE, Moayedi S, Golitaleb M. COVID-19 pandemic hemoperfusion therapy versus plasma exchange therapy in intensive care. Iran J Allergy Asthma Immunol. 2020; 19(S1): 7-9, doi: 10.18502/ ijaai.v19i(s1.r1).2848, indexed in Pubmed: 32534513.

2. Zhai $P$, Ding $Y, W u X$, et al. The epidemiology, diagnosis and treatment of COVID-19. Int J Antimicrob Agents. 2020; 55(5): 105955, doi: 10.1016/j.jjantimicag.2020.105955, indexed in Pubmed: 32234468.

3. Doshmangir L, Ahari AM, Qolipour K, et al. East Asia's strategies for effective response to COVID-19: lessons learned for Iran. Man- 
age Strat Health Syst. 2020; 4(4): 370-373, doi: 10.18502/mshsj. v4i4.2542.

4. Word Health Organization. Coronavirus disease (COVID-19), situation report, 102. Geneva, 2020.

5. Rothan HA, Byrareddy SN. The epidemiology and pathogenesis of coronavirus disease (COVID-19) outbreak. J Autoimmun. 2020; 109: 102433, doi: 10.1016/j.jaut.2020.102433, indexed in Pubmed: 32113704.

6. WuZ, McGoogan JM. Characteristics of and important lessons from the coronavirus disease 2019 (COVID-19) outbreak in China: summary of a report of 72314 cases from the Chinese center for disease control and prevention. JAMA. 2020; 323(13): 1239-1242, doi: 10.1001/ jama.2020.2648, indexed in Pubmed: 32091533.

7. World Health Organization. Coronavirus disease (COVID-19) advice for the public. Geneva, 2020.

8. Noar SM, Zimmerman RS. Health behavior theory and cumulative knowledge regarding health behaviors: are we moving in the right direction? Health Educ Res. 2005; 20(3): 275-290, doi: 10.1093/her/ cyg113, indexed in Pubmed: 15632099.

9. Kakemam E, Ghoddoosi-Nejad D, Chegini Z, et al. Knowledge, attitudes, and practices among the general population during COVID-19 outbreak in Iran: a national cross-sectional online survey. Front Public Health. 2020; 8: 585302, doi: 10.3389/fpubh.2020.585302, indexed in Pubmed: 33363083.

10. Shahabi N, Kamalzadeh Takhti H, Hassani Azad M, et al. Knowledge, attitude, and preventive behaviors of Hormozgan residents toward COVID-19, one month after the epidemic in Iran. Z Gesundh Wiss. 2021 [Epub ahead of print]: 1-12, doi: 10.1007/s10389-020-01454-1, indexed in Pubmed: 33432291.

11. Zhong BL, Luo W, Li HM, et al. Knowledge, attitudes, and practices towards COVID-19 among Chinese residents during the rapid rise period of the COVID-19 outbreak: a quick online cross-sectional survey. Int J Biol Sci. 2020; 16(10): 1745-1752, doi: 10.7150/ijbs.45221, indexed in Pubmed: 32226294.

12. Adab Z, Azizi M, Solhi M, et al. Dormitory student's knowledge and atittiude about influenza a (H1N1) disease. Rahavard Salamat J. 2016; 2: 10-17.

13. McEachan $\mathrm{R}$, Taylor $\mathrm{N}$, Harrison $\mathrm{R}$, et al. Meta-analysis of the reasoned action approach (RAA) to understanding health behaviors. Ann Behav Med. 2016; 50(4): 592-612, doi: 10.1007/s12160-016-9798-4, indexed in Pubmed: 27169555.

14. Moher D, Liberati A, Tetzlaff J, et al. PRISMA Group. Preferred reporting items for systematic reviews and meta-analyses: the PRISMA statement. PLoS Med. 2009; 6(7): e1000097, doi: 10.1371/journal. pmed.1000097, indexed in Pubmed: 19621072.

15. Feleke DG, Alemu Y, Bisetegn $H$, et al. Intestinal parasitic infections and associated factors among street dwellers and prison inmates: A systematic review and meta-analysis. PLoS One. 2021; 16(8): e0255641, doi: 10.1371/journal.pone.0255641, indexed in Pubmed: 34352000.
16. Modesti PA, Reboldi G, Cappuccio FP. Panethnic differences in blood pressure in Europe: A systematic review and meta-analysis. PLoS One. 2016; 11(1): e0147601, doi: 10.1371/journal.pone.0147601.

17. Moradzadeh R, Nazari J, Shamsi M, et al. Knowledge, attitudes, and practices toward coronavirus disease 2019 in the central area of Iran: a population-based study. Front Public Health. 2020; 8: 599007, doi: 10.3389/fpubh.2020.599007, indexed in Pubmed: 33364226.

18. Rahimi M, Maracy MR, Shahraki RA. A survey of knowledge, attitude and practice of the older people about COVID-19 pandemic in Isfahan, Iran. Journal of Gerontology and Geriatrics. 2020; 68(4): 204-211, doi: 10.36150/2499-6564-253.

19. Fallahi A, Mahdavifar N, Ghorbani $A$, et al. Public knowledge, attitude and practice regarding home quarantine to prevent COVID-19 in Sabzevar city, Iran. J Mil Med. 2020; 22(6): 580-588, doi: 10.30491/ JMM.22.6.580.

20. Maharlouei N, Asadi N, Bazrafshan K, et al. Knowledge and attitude regarding COVID-19 among pregnant women in Southwestern Iran in the early period of its outbreak: a cross-sectional study. Am J Trop Med Hyg. 2020; 103(6): 2368-2375, doi: 10.4269/ajtmh.20-0608, indexed in Pubmed: 33124530.

21. Honarvar B, Lankarani KB, Kharmandar A, et al. Knowledge, attitudes, risk perceptions, and practices of adults toward COVID-19: a population and field-based study from Iran. Int J Public Health. 2020; 65(6): 731-739, doi: 10.1007/s00038-020-01406-2, indexed in Pubmed: 32583009.

22. Shaygannejad V, Afshari-Safavi A, Hatef B. Assessment of mental health, knowledge, and attitude of patients with multiple sclerosis and neuromyelitis optica spectrum disorder in response to 2019 novel coronavirus. Neurol Sci. 2021; 42(7): 2891-2901, doi: 10.1007/ s10072-020-04905-5, indexed in Pubmed: 33219424.

23. Nourmoradi $H$, Kazembeigi $F$, Kakaei $H$, et al. Assessment of knowledge, attitude, and practice toward COVID-19 among a sample of Iranian general population. Open Access Macedonian Journal of Medical Sciences. 2020; 8(T1): 167-174, doi: 10.3889/oamjms.2020.5094.

24. Nasirzadeh M, Aligol M. Assessmentof knowledge, attitude, and factors associated with the preventive behaviors of COVID-19 in Qom, Iran, in 2020. Qom Univ Med Sci J. 2020; 14(7): 50-57, doi: 10.29252/qums.14.7.50.

25. Sahraian MA, Gheini MR, Rezaeimanesh N, et al. Knowledge regarding COVID-19 pandemic in patients with multiple sclerosis (MS): a report from Iran. Mult Scler Relat Disord. 2020; 42: 102193, doi: 10.1016/j. msard.2020.102193, indexed in Pubmed: 32420011.

26. Hosseini J, Nematollahi S, Pour ME, et al. Knowledge, attitudes and practice of Tehran citizens regarding the social distancing rules and its related factors during the COVID-19 pandemic. Men's Health Journal. 2020; 4(1): e17, doi: 10.22037/mhj.v4i1.32646.

27. Noori N, Teimouri A, Khalili M, et al. Knowledge, attitude, and practice towards COVID-19 among parents or guardians of patient children. CJHR. 2020; 5(2): 39-44, doi: 10.29252/cjhr.5.2.39. 
28. Kalani N. Knowledge, attitude and practice against the 2019 novel coronavirus (COVID-19) among the Jahrom city people: a cross-sectional study. IJMRA. 2020; 3(9), doi: 10.47191/ijmra/v3-i9-11.

29. Puspitasari IM, Yusuf L, Sinuraya RK, et al. Knowledge, attitude, and practice during the COVID-19 pandemic: a review. J Multidiscip Healthc. 2020; 13: 727-733, doi: 10.2147/JMDH.S265527, indexed in Pubmed: 32801735.

30. Haghighi FK, Kouhi P, Amini M, et al. Knowledge, attitude, and practice toward COVID-19 among healthcare workers in Shiraz, Iran. Shiraz E-Med J. 2020; 21(12): e108872, doi: 10.5812/semj.108872.

31. Pal $R$, Yadav U, Grover $S$, et al. Knowledge, attitudes and practices towards COVID-19 among young adults with type 1 diabetes mellitus amid the nationwide lockdown in India: a cross-sectional survey. Diabetes Res Clin Pract. 2020; 166: 108344, doi: 10.1016/j. diabres.2020.108344, indexed in Pubmed: 32710997.

32. Ajay K, Hamza I, Deepika K, et al. Knowledge \& awareness about COVID-19 and the practice of respiratory hygiene and other preven- tive measures among patients with diabetes mellitus in Pakistan. European Scientific Journal ESJ. 2020; 16(12): 53, doi: 10.19044/ esj.2020.v16n12p53.

33. Melesie Taye G, Bose L, Beressa TB, et al. COVID-19 knowledge, attitudes, and prevention practices among people with hypertension and diabetes mellitus attending public health facilities in Ambo, Ethiopia. Infect Drug Resist. 2020; 13: 4203-4214, doi: 10.2147//DR.S283999, indexed in Pubmed: 33262615.

34. Alzoubi H, Alnawaiseh N, Al-Mnayyis A, et al. COVID-19 - knowledge, attitude and practice among medical and non-medical university students in Jordan. J Pure Appl Microbiol. 2020; 14(1): 17-24, doi: 10.22207/jpam.14.1.04.

35. Chan AKM, Nickson CP, Rudolph JW. Social media for rapid knowledge dissemination: early experience from the COVID-19 pandemic. Anaesthesia. 2020; 75(12): 1579-1582, doi: 10.1111/anae.15057, indexed in Pubmed: 32227594. 